\title{
Neurometry and Neurocraniometry of the Nigerian Local Dog (Canis Iupus familiaris)
}

\section{Igado, Olumayowa Olawumi}

Department of Veterinary Anatomy, Faculty of Veterinary Medicine, University of Ibadan, Nigeria. mayowaigado@yahoo.com

\section{Abstract}

This study investigates the linear morphometry of the brain and the neurocranium which houses it, in the Nigerian local dog. A total of twenty-five dogs (11 males, 14 females) were used for this study. The males recorded a heavier body weight, weight of head and brain. The females recorded higher values for the length of brain, height and length of cerebellum, and the whole skull height and length relative to the males, although no statistically significant differences were observed $(P>0.05)$. Values obtained for the neurocranial volume was similar between the sexes, while the neurocranial height and length was higher in the males, even though no statistically significant differences were observed $(P>0.05)$. Pearson's correlation analysis showed a negative correlation between the weight of the animal and length of brain and the height and length of cerebellum, showing that values for these parameters were likely to relatively higher in younger animals. This study shows correlation relationship between neurometric and neurocraniometric parameters, data obtained may find application in veterinary clinical neuroanatomy of dolichocephalic-type dogs.

\section{Key words}

Nigerian local dog, brain, neurocranium, morphometry, correlation.

\section{Introduction}

The cranium serves as a means of protection for the encephalon and the organs of the special senses (vision, smell, audition, balance and gustation) (Getty, 1986). It consists of a mosaic of many bones that are perfectly fitted together to form a single rigid structure. The cranium is 
divided into two portions, the caudal part (neurocranium) housing the encephalon, while the rostral part sustains the face. In most domestic animals, the face has been observed to be larger than the neural part (Dyce et al., 2000). The interior of the neurocranium presents contours that correspond to the gyri and sulci of the brain; it also corresponds to the space filled by the brain, the meninges and the cerebrospinal fluid in the cranial cavity (Dyce et al., 2000). The bones making up the neurocranium include the occipital, parietal, interparietal, basisphenoid, pterygoid, temporal, frontal, ethmoid and the vomer (Getty, 1986). The shapes of the skull in dogs show considerable variation in breed and size, and so is the most important criterion in determining the standard breed of dogs (Onar et al., 2002).

The Nigerian local dogs (NLD) are a breed indigenous to Nigeria; they are popularly referred to as 'mongrels' by indigenes. They are dolichocephalic (long-headed) dogs and are highly domesticated, with their feeding pattern being majorly omnivorous due to the high level of domestication. A lot of data has been generated on the haematological, biochemical and parasitological profile of this breed of dog (Awah and Nottidge, 1998; Ariyibi et al., 2002; Olayemi et al., 2009), but there is presently a dearth of information on the gross morphological appearance of this breed of dog. This study aims to determine the linear neurometry and neurocraniometry of this breed relative to other species of dogs studied.

\section{Materials and Methods}

Ethical approval for this study was obtained from the Ethical Committee of the Faculty of Veterinary Medicine, University of Ibadan, Nigeria, Ethical code number 'ethic/05/11/01'. All procedures followed the Guide for the care and use of experimental animals (Faculty of Veterinary Medicine, University of Ibadan, Nigeria).

A total of twenty-five clinically healthy adult Nigerian local dogs (11 males, 14 females) were used for this study. All animals were aged 2 years or more. Age was obtained from vendors and confirmed using the dental formula according to Dyce et al., (2002). The animals were weighed with a standard bathroom scale and euthanized by intravenous injection of pentobarbitone. The heads were severed at the atlanto-occipital junction and weighed. The brain was removed by the following procedure: a sharp scalpel 
blade was used to cut the skin dorsally, in a mid-sagittal plane, from the nasal region to the base of the skull, at the region of the foramen magnum. The skin was reflected to expose the bone. An industrial saw (band-saw: Startrite 24-S-5, Eng. Co. Ltd, England) was used to cut the skull in a mid-sagittal plane, thereby exposing the brain. Linear measurements were taken on the brain in-situ, after which all brain material were removed and weighed with a digital weighing scale. The skull was de-fleshed with a scalpel blade as much as possible, and subsequently subjected to a modification of the hot water maceration technique as previously described by Onar (1999). Briefly, the heads were tagged and labelled for ease of identification, then placed in water containing polycarboxylate and anionic surfactant (detergent), with soap chips (sodium carbonate), heated to $100^{\circ} \mathrm{C}$ for one hour. Further removal of muscle and ligament was done, and the skulls were rinsed with clean water and thereafter transferred to a water solution containing detergent, $0.3 \%$ sodium hypochlorite and $6 \%$ hydrogen peroxide solution for 18 hours. The skulls were again rinsed in clean water and then left in the sun to dry.

A total of 20 parameters were determined on the brain and skull, some illustrated in figures 1-2. Linear measurements were made to the nearest $0.01 \mathrm{~mm}$, and determined with the aid of vernier callipers. Parameters measured and their landmarks are defined below:

Weight of animal (WOA): This was determined using a standard bathroom scale. Measurements were recorded in kilograms.

Weight of head (WOH): Measured with the aid of a "Five goat" spring scale $\AA$. Measurements were recorded in kilograms.

Weight of brain (WOB): this was determined with the aid of digital weighing scale (Microwa swiss balance ${ }$, Mettler-Toledo, Switzerland) and recorded in grams.

Length of Brain (LOB): Measured from the most rostral point of the olfactory bulb to the medulla oblongata.

Height of Brain (HOB): Distance from the dorsal aspect of the cerebrum to the ventral aspect of the brainstem.

Height of Cerebrum (HOC): From the dorsal aspect of the cerebrum to the dorsal aspect of the brainstem.

Length of Cerebrum (LOC): Distance from the rostral pole of the cerebrum to its most caudal pole.

Height of Cerebellum (HOCB): Measured from the highest point of the median vermis to the roof of the fourth ventricle. 
Length of Cerebellum (LOCB): Measured from the most rostral end of the cerebellum as the cerebellum makes contact with the cerebrum, to the most caudal point or far extremity, rostral to the medulla oblongata.

Neurocranial volume (NCV): this was achieved by using cotton wool to block all the foramina of the intact skull, fine grains was then poured into the neurocranium via the foramen magnum until filled up. The grains were then emptied into a measuring cylinder and the volume was determined.

Neurocranial Height (NCH): this was the distance, measured as a straight line, from the roof of the neurocranium (frontal bone) to the floor (sella turcica of the sphenoid bone). (Fig 1).

Neurocranial Length (NCL): distance from the deepest indentation of the fronto-ethmoidal bone, to the caudal aspect of the cerebral surface of the occipital bone, at the level of the external occipital protuberance.

Neurocranial Index (NCl): this was calculated as $\mathrm{NCH} / \mathrm{NCL} \times 100$

Whole Skull Height (WSH): the height of the skull with the mandible, taken as the distance from the most dorsal aspect of the frontal bone, to the most ventral aspect of the mandible. (Fig 2).

Whole Skull Length (WSL): this was taken as the length of the skull, from the most rostral aspect of the incisive bone to the most caudal aspect of the occipital bone. Figure 2 .

Whole Skull Index: this was calculated as WSH/WSL $\times 100$.

Statistical analysis: All data were analysed using Graphpad prism soft ware (V4). Students t' test was carried out at $p<0.05$ level of significance.

\section{Results}

Results are presented in Tables 1-2; absolute values as Mean \pm Standard deviation in Table (1) and Pearson's correlation coefficient values in Table (2).

Of all the sixteen parameters measured, the females were observed to record higher values in only five (length of brain, height and length of cerebellum, whole skull height and length), although no statistically significant difference was observed $(P>0.05)$. Statistically significant difference between the genders was only observed in the height of the cerebrum $(P<0.05)$ (Table 1$)$. The males recorded heavier body, head and brain weights (males 15.75 $\pm 6.19 \mathrm{~kg}, 1.05 \pm 0.24 \mathrm{~kg}$ and 53.50 \pm 3.54 grams respectively; females $14.11 \pm 4.26 \mathrm{~kg}, 0.95 \pm 0.28 \mathrm{~kg}, 49.64$ $\pm 10.41 \mathrm{~g}$ respectively), but the rela- 
tive brain weight $(0.004 \%)$ was observed to be higher in the females $(0.004 \%)$ relative to the males $(0.003 \%)$. Correlative analysis of the weight of the animal with the other parameters measured gave positive values (ranging from no correlation to medium correlation) except the length of brain, and the height and length of the cerebellum $(r=-0.048$, -0.537 and -0.132 respectively). The neurocranial volume showed the highest positive correlation to the body weight $(r=0.587)$, followed by the weight of the head $(r=0.553)$, while the length of the brain showed the lowest and most negligible negative correlation $(r=-0.048)$ while the height of the cerebellum showed the highest negative correlation $(r=-0.537)$ (Table 2).

\section{Discussion}

While there is information on the measurement of the brain and skulls of other breeds of dogs, adult and puppy, there is presently a dearth on information concerning the NLD. For this purpose, neurometric and neurocraniometric measurements were investigated. This study shows the correlative relationship between the brain and the neurocranium.

The higher body weight observed in the males is in consonance with the observation of Campbell (1990) that males usually have a higher body weight relative to females of the same age and species. The relative brain weight of $0.004 \%(1: 271)$ obtained is similar to that obtained in pigs (Olopade et al., 2011), lower than the $1: 100$ and 1:125 obtained in other dogs and cats respectively, but higher than the 1:550, 1:560 and 1:600 obtained in the lion, elephant and horse respectively (Kuhlenbeck, 1973).

In spite of the fact that the males recorded higher values for the brain weight and height, the females recorded slightly higher values for the length of the brain. This difference may be attributed to the longer length of cerebellum in the females and probably a longer olfactory bulb. The heavier brain weight recorded in the males is similar to what was obtained in pigs (Olopade et al., 2011), although the male pigs recorded higher values for brain length while the females recorded higher values for brain height; no statistically significant differences were observed $(P>0.05)$. The cerebellar parameters were observed to be higher in the females, although no statistically significant difference was observed $(P>0.05)$. This observation is at variance with what obtains in humans, using Korean adults, where the males were ob- 
served to have a statistically significant larger cerebellar volume (Rhyu et al., 1999). This observation in the NLD may indicate that the females of this breed are more intelligent, may have finer motor dexterity and also possess better cognitive ability, as cerebellar volume has been shown to have a positive correlation to general intelligence and fine motor dexterity; it also contributes to several aspects of cognition (Paradiso et al., 1997).

The neurocranial volume maintained a negligible to mild positive correlation with all the parameters determined except the height and length of the cerebellum and the neurocranial index $(r=-0.242$, 0.502 and -0.123 respectively). Since the neurocranial volume displayed a positive correlation to the body weight, the negative correlations observed between the neurocranial volume and cerebellar length may indicate that the younger animals (of smaller body weight) may have a relatively larger cerebellar/neurocranial volume ratio when compared with adults. This negative correlation between the weight of the animal and height of the cerebellum is similar to a recent report in the pig (Olopade et al., 2011). The highest positive neurocranial volume correlations were observed with the heights of the brain and cerebrum ( $r=0.785$ and 0.736 respectively).

In this study, the high positive correlation observed between the neurocranial volume and neurocranial height, and a lesser one between the volume and length is similar to what was reported by Garcia-Real et al. (2004) in a study conducted on live dogs of different skull shapes (brachycephalic, mesaticephalic and dolichocephalic dogs), using the computerised tomographic method. A positive correlation between the neurocranial volume and the height and length has also been reported in humans (Acer et al., 2006). Also, Garcia-Real et al. (2004) in the previously mentioned study recorded a significantly higher neurocranial volume in the males relative to the females. Rodrigues et al. (2010) also recorded a higher value for the neurocranial volume in male goats relative to females, although no statistically significant difference was observed. This differs from what was observed in this study where the values obtained were very similar, thereby showing no sexual dimorphism. This similarity between the sexes is however not reflected in the linear measurements determined. The similar neurocranial volume and differing linear measurements between the genders can imply that the males are more prone to 
increased intra-cranial pressure, since they recorded higher values for the brain height and weight. The slight or mild positive correlation observed between the whole skull length and the neurocranial length may be considered to be similar to earlier reports in the Alsatian puppies where positive correlations, although high and significant, were also reported (Onar, 1999). In addition, the neurocranial volume displayed a slightly negative correlation with the neurocranial index.

In conclusion, the morphometric analysis of the brain and its comparison to the cranial cavity parameters, as highlighted by this study, will facilitate the detection of pathologies such as cerebral atrophy (Onar et al., 2002). Data obtained from this investigation may be applicable in the field of veterinary clinical neuroanatomy, comparative and regional anatomy; it may also make it possible for future researchers to better understand the dolichocephalic breed of dogs, using the NLD as a prototype.

\section{Acknowledgements}

The author is grateful to Mr A.W. Ramoni and Dr. O.K. Ekeolu, both of the Department of Veterinary
Anatomy, University of Ibadan, for their technical assistance.

\section{References}

Acer, N, Sahin, B, Ekinci, N, Ergür, $\mathrm{H}$, Basaloglu, H. (2006): Relation between intracranial volume and the surface area of the foramen magnum. The Journal of Craniofacial Surgery, 17, 326-330.

Ariyibi, A.A, Oyeyemi, M.O, Ajadi, R.A. (2002): Comparative study of some haematological and biochemical parameters of clinically healthy Alsatian and local dogs. African Journal of Biome-dical Research, 5, 145-147.

Awah, J.N and Nottidge, H.O. (1998): Serum biochemical parameters in clinically healthy dogs in Ibadan. Tropical Veterinarian, 16:123-129.

Campbell, N.A. (1990): Biology. $2^{\text {nd }}$ edition. The Benjamin / Cummings publishing Company Inc, New York, page 229-343.

Dyce, K.M., Sack, W.O, Wensing, C.J.G, Molenaar, G.J. (2000) : Agreement of veteri-nary anatomy. Rio de Janeiro: Elsevier, page 293.

Dyce, K.M, Sack, W.O, Wensing, C.J.G. (2002): Textbook of 
Veterinary Anatomy. $3^{\text {rd }}$ edition, Saunders.

Garcia-Real, I., Kass, P.H, Sturges, B.K, Wisner, E.R. (2004): Morphometric analysis of the cranial cavity and flow cranial fossa in the dog: the computerized tomographic study. Veterinary Radiology \& Ultrasound, 45, 38-45.

Getty, R (1986): General Osteologia. In Getty, R. (Ed.). Sisson/Grossman - Anatomy of the domestic animals. Rio de Janeiro: Guanabara Koogan, p. 29-30. vol. 1.

Kuhlenbeck, H. (1973): Central Nervous System of Vertebrates. Vol. 3, Part II. ArnoldBacklin-Strasse, New York.

Olayemi,F.O,Azeez, I.O, Ogunyemi, A, Ighagbon, F.O. (2009): Study on erythrocyte values on the Nigerian indigenous dog. Folia Veterinaria, 53(2), 65-67.

Onar, V. (1999): A Morphometric Study on the Skull of the German Shepherd Dog "Alsatian". Anat. Histol. Embryol. 28, 253-256.

Onar, V, Kahveciodlu, K.O, Cebi, V. (2002): Computed tomogramphic analysis of the cranial cavity and neurocranium in the German Shepherd dog (Alsatian) puppies.
Veterinarski Arhiv, 72(2), 5766.

Olopade, J.O, Igado, O.O, Nwafor, C.I, Alamu, A.O, Onwuka, S.K. (2011): Some aspects of the craniofacial indices and macro neurometrics of the Nigerian local pig (Sus Scrofa). Int. J. Anat. Embryol ., In press.

Paradiso, S., Andreasen, N.C, O'Leary, D.S, Arndt, S, Robinson, R.G. (1997): Cerebellar Size and Cogni-tion: Correlations with IQ, Verbal Memory and Motor Dexterity, Neuropsychiatry Neuropsychology Behaviuo-ral Neurology 10(1), 1-8.

Rodrigues, R.T.S., Matos, W.C.G, Walker, F.M, Costa, F.S, Wanderley C.W.S, Pereira Neto, J, Faria, M.D. (2010): Dimensions of the cranium and of the cranial cavity and intracranial volume in goats (Capra hircus LINNAEUS, 1758). Journal of Morphological Science, 27(1), 6-10.

Rhyu, I.J., Cho, T.H, Lee, N.J, Uhm, $\mathrm{C}, \mathrm{Kim}, \mathrm{H}$, and Suh, $\mathrm{Y}$. (1999): Magnetic resonance image-based cerebellar volumetry in healthy Korean adults. Neuroscience Letters, 270 (3), 149-152. 


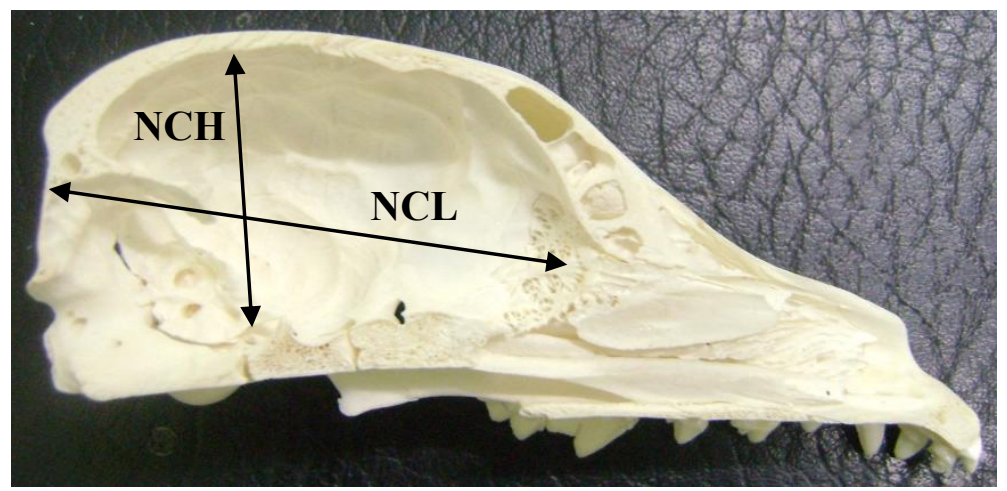

Fig (1): Mid-sagittal section of skull, showing the neurocranial height $(\mathrm{NCH})$ and neurocranial length (NCL).

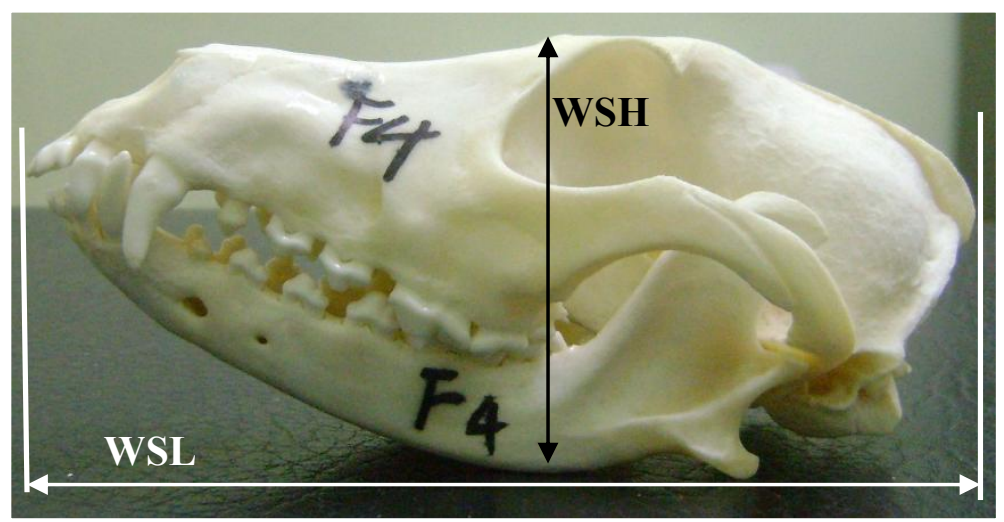

Fig (2): Lateral view of skull showing the Whole Skull Height (WSH) and the Whole Skull Length (WSL). 
Table (1): Neurometrical and neurocraniometrical values of the Nigerian local dog

\begin{tabular}{|c|c|c|c|}
\hline Parameters & Total $n=25$ & Male, $n=11$ & $\begin{array}{c}\text { Female, } n= \\
14\end{array}$ \\
\hline Weight of animal (kg) & $14 \pm 4.44$ & $15.75 \pm 6.19$ & $14.11 \pm 4.26$ \\
\hline Weight of head (kg) & $0.94 \pm 0.25$ & $1.05 \pm 0.24$ & $0.95 \pm 0.28$ \\
\hline Weight of brain (g) & $51.75 \pm 7.33$ & $53.50 \pm 3.54$ & $49.64 \pm 10.41$ \\
\hline Length of brain (cm) & $7.20 \pm 0.43$ & $7.17 \pm 0.19$ & $7.25 \pm 0.64^{\#}$ \\
\hline Height of brain $(\mathrm{cm})$ & $3.97 \pm 0.36$ & $4.08 \pm 0.37$ & $3.84 \pm 0.33$ \\
\hline Height of cerebrum $(\mathrm{cm})$ & $3.25 \pm 0.34$ & $3.48 \pm 0.14^{*}$ & $2.98 \pm 0.30^{*}$ \\
\hline Length of cerebrum $(\mathrm{cm})$ & $4.61 \pm 0.35$ & $4.73 \pm 0.05$ & $4.47 \pm 0.51$ \\
\hline Height of cerebellum $(\mathrm{cm})$ & $1.9 \pm 0.25$ & $1.84 \pm 0.15$ & $1.99 \pm 0.37^{\#}$ \\
\hline Length of cerebellum $(\mathrm{cm})$ & $1.86 \pm 0.31$ & $1.73 \pm 0.29$ & $2.06 \pm 0.23^{\#}$ \\
\hline Neurocranial volume (ml) & $60 \pm 9.49$ & $60 \pm 5.72$ & $60 \pm 12.47$ \\
\hline Neurocranial height (cm) & $3.82 \pm 0.29$ & $3.88 \pm 0.23$ & $3.78 \pm 0.33$ \\
\hline Neurocranial length $(\mathrm{cm})$ & $7.16 \pm 0.64$ & $7.17 \pm 0.22$ & $7.14 \pm 0.86$ \\
\hline Neurocranial index (\%) & $54 \pm 3.24$ & $54.11 \pm 2.10$ & $53.9 \pm 4.18$ \\
\hline Whole skull height (cm) & $8.13 \pm 0.85$ & $7.88 \pm 0.62$ & $8.31 \pm 0.99^{\#}$ \\
\hline Whole skull length $(\mathrm{cm})$ & $16.39 \pm 1.77$ & $15.8 \pm 1.1$ & $16.76 \pm 2.07^{\#}$ \\
\hline Whole skull index (\%) & $50.07 \pm 1.55$ & $50.77 \pm 1.07$ & $49.64 \pm 1.70$ \\
\hline
\end{tabular}

*Indicates statistically significant difference $(P<0.05)$ between male and female values, observed only in the height of the cerebrum.

\# Indicates values which were observed to be higher in female dogs, although no statistically significant differences were observed $(P>0.05)$. 


\begin{tabular}{|c|c|c|c|c|c|c|c|c|c|c|c|c|c|}
\hline & $\begin{array}{l}f \\
0 \\
0 \\
0 \\
0 \\
0 \\
0\end{array}$ & 永 & & & & & & & & & & & \\
\hline 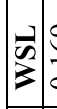 & 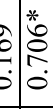 & & & & & & $\begin{array}{l}\hat{2} \\
\vdots \\
0 \\
0\end{array}$ & & & & & & \\
\hline i & & & & & i & & & & & & & & \\
\hline & & & & & $\begin{array}{l}v \\
0 \\
0\end{array}$ & & $\begin{array}{l}\vec{\Xi} \\
\overrightarrow{0} \\
\dot{0}\end{array}$ & $\vec{Z}$ & & & & & \\
\hline 40 & & & & & $c$ & & 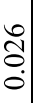 & $\stackrel{f}{*}$ & & & & & \\
\hline$z$ & & & & & i & & $\begin{array}{l}n \\
\vdots \\
0 \\
0\end{array}$ & $\stackrel{*}{*}$ & & & & & \\
\hline & $\stackrel{0}{0}$ & & & & 10 & & $\begin{array}{l}0 \\
\vdots \\
\vdots \\
\vdots \\
1\end{array}$ & & & & & & \\
\hline בِ & $\begin{array}{l}1 \\
\\
1\end{array}$ & & 0 & & & & & & & & & & \\
\hline
\end{tabular}

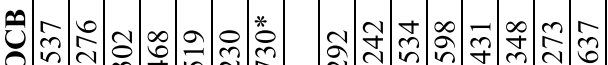

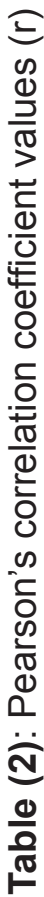

ำ

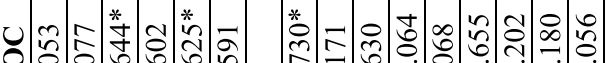

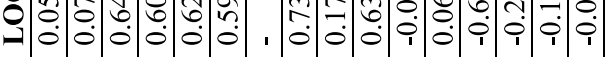

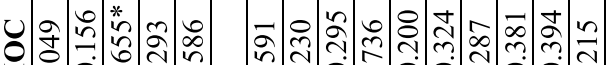
I

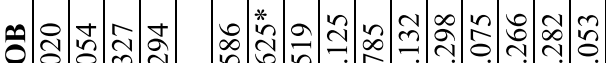

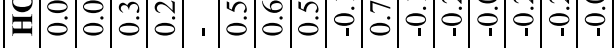

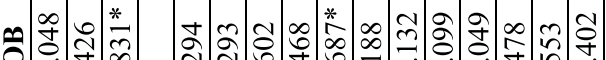

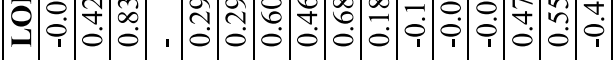
๐ี

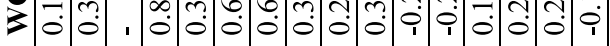

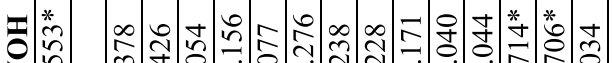

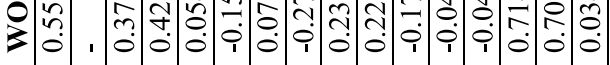

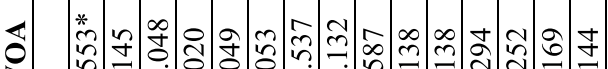

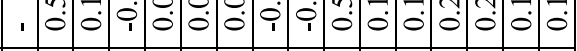

